\title{
Tratamiento Quirúrgico de la Recesión en el Sector Anterior a través de la Técnica de Túnel Modificada Utilizando Matriz Dérmica Acelular.
}

\author{
Surgical Treatment of the Recession in the Anterior Sector \\ through the Modified Tunnel Technique Using the Acellular Dermal \\ Matrix.
}

Israel Antonio Juárez-Membreño ${ }^{1^{*}}$

\begin{abstract}
1. Periodoncista. Cátedra de Periodoncia. Instituto de Odontoestomatología. Escuela de Odontología. Facultad de Medicina. Universidad Austral de Chile. Valdivia-Chile.
\end{abstract}

* Correspondencia autor. Israel Antonio JuárezMembreño | Dirección: Rudloff 1640 Valdivia | Telefono: 56632293751 | E-mail: juarezuach@ gmail.com

Trabajo recibido el 24/07/2018.

Aprobado para su publicación el 22/12/2018

\begin{abstract}
RESUMEN
Objetivo: El propósito del reporte de caso fue evaluar la cobertura radicular (CR) en el sector anterior luego de haber realizado la técnica de túnel modificada utilizando Matriz Dérmica Acelular (MDA). Consideraciones clínicas: La literatura ha descrito el uso de la técnica de túnel desde hace varias décadas y en los últimos años se ha perfeccionado con desplazamiento del tejido gingival hacia coronal simultáneo con el uso de MDA con el fin de lograr mejores resultados. Aunque en situaciones clínicas de cobertura de recesión gingival (RG) es discutible qué técnica puede ser más efectiva y existen diferentes alternativas, la técnica de túnel más colgajo desplazado coronal utilizando MDA es una línea de investigación prometedora y todavía no está concluída por tanto sería interesante profundizar al respecto. Conclusión: El caso clínico presenta el seguimiento de un paciente con recesión tipo 1 (RT1), que fue operado utilizando MDA y la técnica de túnel modificada demostrando que puede ser efectiva en la cobertura total de la recesión con resultados estéticos sostenibles en un período de 24 meses.
\end{abstract}

PALABRAS CLAVE:

Matriz dérmica acelular, Recesión gingival.

Rev. Clin. Periodoncia Implantol. Rehabil. Oral Vol. 12(2); 103-105, 2019.

\begin{abstract}
Objective: The purpose of the case report was to evaluate the radicular coverage $(R C)$ in the upper anterior sector after having performed the modified tunnel technique using the Acellular Dermal Matrix (ADM). Clinical considerations: The literature has described the use of the tunnel technique for several decades and in recent years has been perfected with displacement of the gingival tissue to coronal with the use of ADM to achieve better results. Although in clinical situations of gingival recession (GR) coverage it is questionable which technique can be more effective and there are different alternatives, the tunneling technique plus coronal displacement flap using ADM is a promising line of research and is not yet concluded so it would be interesting to deepen in this regard. Conclusion: The clinical case presents the follow-up of a patient with recession type 1 (RT1) who was operated using ADM and the modified tunnel technique demonstrating that it can be effective in the total coverage of the recession with sustainable aesthetic results in a period of 24 months.
\end{abstract}

KEY WORDS:

Acellular dermal matrix, Gingival recession.

Rev. Clin. Periodoncia Implantol. Rehabil. Oral Vol. 12(2); 103-105, 2019

\section{SIGNIFICANCIA CLÍNICA}

El estudio del uso de matriz dérmica acelular a través de la técnica de túnel modificada es una línea de investigación que es interesante profundizar ya que puede ser una opción efectiva en odontología estética sobre todo cuando se desea corregir la recesión gingival individual en el sector anterior, tomando en cuenta la disminución de la morbilidad de los tejidos periodontales y efectividad al usar solo un sitio quirúrgico.

\section{INTRODUCCIÓN}

La cirugía plástica periodontal involucra procedimientos quirúrgicos que tienen el objetivo de prevenir, corregir, eliminar las deformidades de la mucosa alveolar como la morfología, posición, dimensión de la encía y tejido óseo que rodean al diente o al implante. Una de las indicaciones más comunes es el tratamiento de la recesión gingival (RG), que es la exposición radicular debido a la migración de los tejidos periodontales 
hacia apical de la unión cemento esmalte, puede ser localizada o generalizada con una o más superficies comprometidas y su etiología está asociada a factores anatómicos, fisiológicos y patológicos ${ }^{(1,2)}$.

El tratamiento de RG se ha convertido en un problema terapéutico debido al aumento de las demandas estéticas y funcionales de los pacientes ${ }^{(3-6)}$. Existen técnicas quirúrgicas para tratar áreas de RG y aumentar zona de tejido como: injerto gingival libre, colgajo posicionado lateralmente, colgajo de doble papila, colgajo semilunar, colgajo desplazado coronal, injerto de tejido conectivo subepitelial (SCTG), regeneración tisular guiada y el uso de aloinjertos de tejidos acelulares $^{(1,2-4)}$. El SCTG con desplazamiento coronal es predecible, sin embargo necesita un segundo sitio quirúrgico con riesgo de dolor postoperatorio o insuficiente cantidad de tejido autógeno(2).

La matriz dérmica acelular (MDA), un aloinjerto dérmico procesado para extraer los componentes celulares trabaja similar a un injerto autógeno, proporciona matriz bioactiva de colágeno, elastina, canales de vasos sanguíneos, proteínas bioactivas ${ }^{(1,2,4,5,7,8)}$. La capa dérmica se lava en soluciones detergentes para inactivar virus, reducir rechazo. Se crioprotege, liofiliza rápidamente en un proceso patentado para preservar su integridad bioquímica estructural. Actúa como un andamiaje de células endoteliales vasculares y logra que los fibroblastos colonicen la matriz ${ }^{(1,2-4,9-11)}$. Aumenta el espesor, se adhiere a los tejidos periodontales similar al tejido conectivo. Sus cualidades no inmunogénicas hacen que sea viable el trasplante dermal, manteniendo integridad estructural y revascularización ${ }^{(2,4)}$.

Su uso es a través de colgajo posicionado coronal y técnica de túnel(1). La técnica de túnel como procedimiento para cobertura radicular fue introducido en 1994 con característica de preservar la papila intacta, tendencia a mejor estética. Probablemente su principal ventaja es que puede producir menor molestia en zona receptora y es un procedimiento mínimamente invasivo sin necesidad de obtener tejido palatino ${ }^{(2,7,10)}$.

Se modificó la técnica para incluir colocación coronal de tejido marginal. A través de disección más profunda se libera tejido gingival vestibular y mediante el levantamiento desde el interior de las papilas permite movilización en el desplazamiento del colgajo alcanzando cobertura completa ${ }^{(2,6,10)}$. El propósito del reporte de caso es evaluar la $\mathrm{CR}$ en el sector anterior luego de haber realizado la técnica de túnel modificada y MDA.

\section{PRESENTACIÓN DEL CASO}

Paciente de sexo femenino, 22 años de edad, chilena, sin enfermedades sistémicas, no fumadora. Acude a Especialidad de Periodoncia ya que desea efectuar CR en el sector anterior. Al examen clínico presenta en el canino superior izquierdo (Fig.1): PSR código cero, profundidad de surco $2 \mathrm{~mm}$, ausencia de signos y síntomas de inflamación, control de higiene adecuado, suficiente encía queratinizada, recesión gingival tipo $1 \mathrm{RT} 1^{(11)}$ ya que al indagar en la anamnesis la recesión está asociada con el cepillado dental traumático en tejido periodontal sano y la recesión solo se extiende 5 milímetros de la unión cemento esmalte en superficie vestibular (Fig.2). Es importante aclarar que el factor etiológico fue corregido antes del procedimiento ya que es parte fundamental para el éxito del tratamiento.

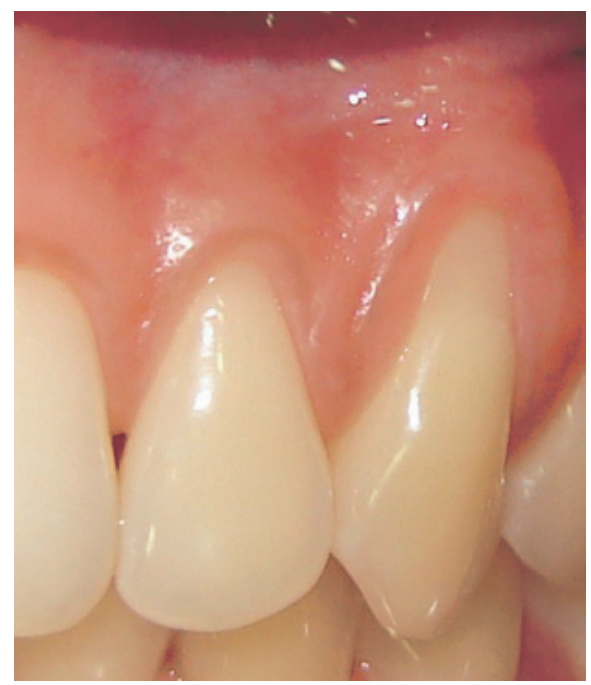

Figura 1. Evaluación preliminar. Recesión tipo 1 de Cairo en canino superior izquierdo

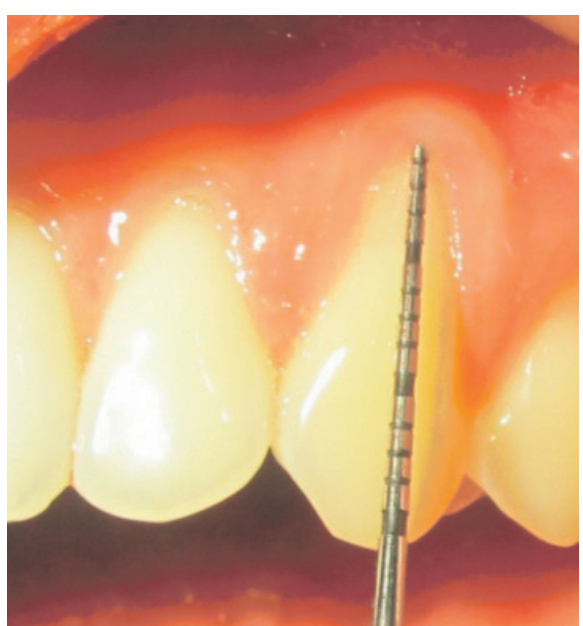

Figura 2. Extensión de la recesión

\section{Procedimiento quirúrgico y resultado clínico}

Se efectúa anestesia local (mepivacaína 2\%, epinefrina 1:100,000), luego se procede a realizar técnica de túnel modificada en canino superior izquierdo, utilizando el instrumental de cirugía plástica periodontal ( $1 / 2$ bisturí Allen Orban para tunelización, perióstotomo anterior con extremo doble, portaguja castroviejo), matriz dérmica acelular ALLODERM. Se realiza incisión intracrevicular sin descargas verticales, desde el interior desplazamiento parcial del tejido periodontal conservando el periostio y la integridad de la papila interdentaria (Fig. 3). La extensión es a partir del diente adyacente con el objetivo de introducir la matriz y lograr un adecuado manejo de los tejidos.

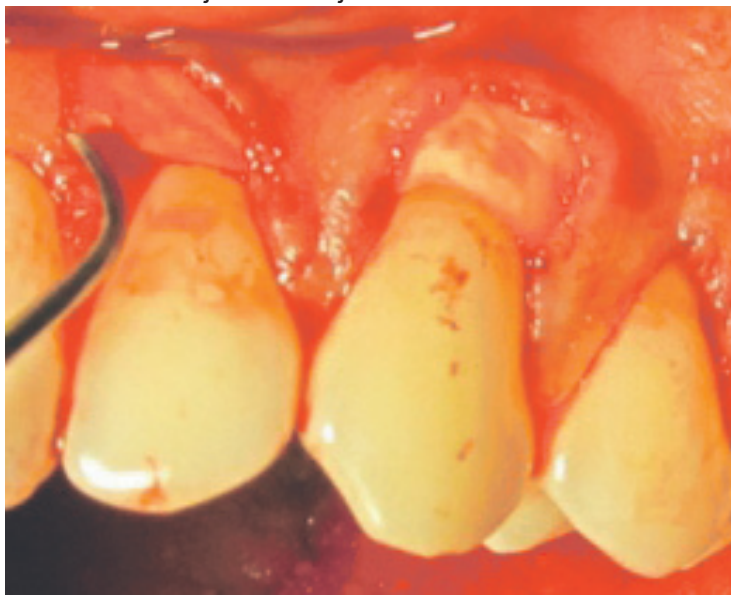

Figura 3. Introducción de la MDA en el sitio quirúrgico de la recesión. Existe ausencia de incisiones verticales e integridad de la papila gingival

Se usa sutura reabsorbible polysorb 6-0* lactomer 9-1, (0.7 metric) $45 \mathrm{~cm}$ SL-5688. Finalmente a través de puntos simples y suspensorios, material no reabsorbible Mononylon Ethilon 3-0 (2 metric) $75 \mathrm{~cm}$ triangular SC-24, se lleva el tejido hacia coronal para lograr la modificación de la técnica de túnel obteniendo cobertura completa de la MDA (Fig. 4). Se indica ketoprofeno $200 \mathrm{mg} 1$ cada 24 horas por 3 días, enjuague de clorhexidina $0.12 \%, 15 \mathrm{ml}$ por 60 segundos dos veces al día por 5 días. Control a los 5 días descartando complicaciones y el retiro de sutura es realizado dos semanas después de la cirugía.

Con respecto al resultado clínico se obtuvo cobertura total de la recesión, ausencia de inflamación, profundidad al sondaje de 2 milímetros, posición de encía a nivel de la unión cemento esmalte y aumento de espesor de tejido queratinizado durante 24 meses posterior a la cirugía (Fig. 5).

\section{DISCUSIÓN}

En tratamiento quirúrgico de RG es discutible cual técnica puede ser más efectiva. El caso clínico de un paciente con recesión tipo $1^{(11)}$ que fue operado utilizando MDA y técnica de túnel modificada logró cobertura total con resultados estéticos sostenibles en un período de 24 meses.

Al aplicar el sistema de códigos estéticos que evaúa cobertura 


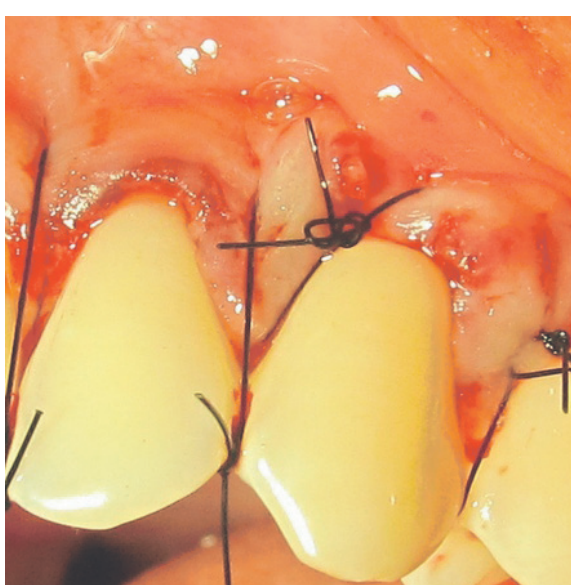

Figura 4. Desplazamiento coronal del colgajo después de colocar la MDA

radicular(12) existió puntaje favorable y sostenido en el tiempo sobre posición de margen gingival, contorno de los tejidos, textura, alineamiento con respecto a posición de línea mucogingival y logro de

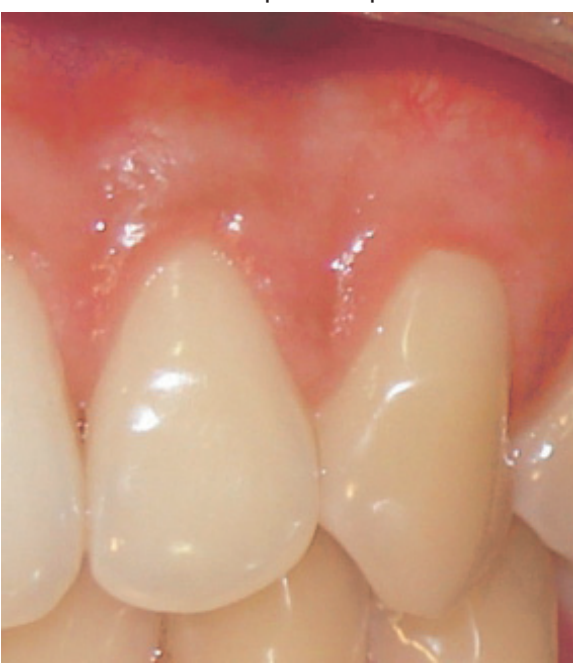

Figura 5. Control 24 meses después de la cirugía periodontal. Existe cobertura total de la recesión color natural,ventaja de la técnica descrita. (Fig. 5)

Aunque la técnica parece ser exitosa, hay limitaciones como el grado de cobertura de raíz que está asociado con altura de hueso interproximal disponible para fijación gingival, además se ha descrito contraindicación en casos de biotipo gingival fino y ausencia de mucosa queratinizada punto clave para el éxito del caso clínico presentado donde el espesor de tejido periodontal era favorable. Éxito en ausencia de mucosa queratinizada al usar MDA es crítico, la evidencia sugiere evaluaciones a largo plazo(13).

Al comparar el uso de colgajo posicionado coronal solo versus colgajo posicionado coronal más MDA, los resultados han demostrado que el colgajo posicionado coronal logra $78 \%$ de cobertura y colgajo en posición coronal más MDA puede alcanzar casi $100 \%^{(14)}$ similar al caso clínico donde la cobertura de recesión fue 100\% y estable durante 24 meses.

Otro aspecto es la modificación de túnel a través del desplazamiento coronal de tejido, la evidencia clínica sostiene que es efectivo y recomendable el desplazamiento coronal(15). Según el resultado del caso clínico se sugiere acompañar el desplazamiento coronal en la técnica de túnel.

Con respecto al tiempo que se puede mantener la cobertura de RG. La evidencia indica que es fundamental el seguimiento de los casos para demostrar si es sostenible en períodos $\operatorname{largos}^{(2)}$. Se ha comprobado que en un año el uso de MDA logra CR total tanto en ancho y largo de la recesión ${ }^{(14)}$ además existió otra investigación que demostró que MDA puede reducir RG y ganar mayor espesor gingival después de 24 meses a través de la modificación del colgajo coronal(5) similar con el caso, donde la posición del margen gingival se mantuvo sobre la línea de unión cemento-esmalte por un período de 24 meses.

Esta línea de investigación es prometedora y todavía se puede profundizar, la perspectiva futura nos debe impulsar a realizar investigaciones con una muestra mayor de pacientes y seguimiento a largo plazo.

\section{CONCLUSIÓN}

El caso clínico demostró cobertura completa en RG utilizando MDA y la técnica de túnel modificada en el sector anterior superior. No obstante un clínico no debe olvidar que cada caso es diferente, es necesario evaluar las dimensiones y espesor del tejido periodontal, el tipo y tamaño de la recesión gingival de cada situación.

\section{CONFLICTO DE INTERÉS}

El autor declara no presentar conflicto de interés.

\section{Bibliografía}

1. Taylor J, Gerlach R, Herold R, Bisch F, Dixon D. A modified tensionless gingival grafting technique using acellular dermal matrix. Int J Periodontics Restorative Dent. 2010;30:513-21.

2. Juarez Membreño I. Comparación de dos técnicas en el manejo quirúrgico de la recesión radicular utilizando matriz dérmica acelular. Reporte de un caso. Av. Odontoestomatol. 2014;30:187-94.

3. Mahn D. Esthetic correction of gingival recession using a modified tunnel technique and an acellular dermal connective tissue allograft. J Esthet Restor Dent. 2002;14:18-23.

4. Andrade P, Felipe M, Novaes A Jr., Souza S, Taba M Jr., Palioto D, et al. Comparison between two surgical techniques for root coverage with an acellular dermal matrix graft. J Clin Periodontol. 2008;35:263-269.

5. de Queiroz A, Sallum AW, Casati MZ, Nociti HF, Salum EA. A two-year prospective study of coronally positioned flap with or without acellular dermal matrix graft. J Clin Periodontol. 2006;33:683-9.

6. Cairo F, Pagliaro U, Buti J, Baccini M, Graziani F, Tonelli P, et al. Root coverage procedures improve patient aesthetics. A systematic review and Bayesian network meta-analysis. J Clin Periodontol. 2016;43:965-975.

7. Modarressi $M$, Wang $H$. Tunneling procedure for root coverage using acellular dermal matrix: A case series. Int J Periodontics Restorative Dent. 2009;29:395-3. 8. Allen EP, Swift EJ. Crown margins in the esthetic zone. J Esthet Restor Dent. 2011;23:57-60
9. Barros R, Novaes AB, Grisi M, Souza S, Taba M, Palioto D. New surgical approach for root coverage of localized gingival recession with acellular dermal matrix: A 12-Month Comparative Clinical Study. J Esthet Restor Dent. 2005; 17:156-164

10. Zucchelli G, Mounsiff I. Periodontal plastic surgery. Periodontol 2000. 2015; 68:333-368.

11. Cairo F, Nieri M, Cincinelli S, Mervelt J, Pagliaro U. The interproximal clinical attachment level to classify gingival recessions and predict root coverage outcomes: an explorative and reliability study. J Clin Periodontol. 2011; 38:661-666.

12. Cairo F, Rotundo R, Miller PD, Pini Prato GP. Root coverage esthetic score: a system to evaluate the esthetic outcome of the treatment of gingival recession through evaluation of clinical cases. J Periodontol. 2009; 80:705-710.

13. Kim DM, Neiva R. Periodontal soft tissue non-root coverage procedures: A systematic review from the AAP Regeneration Workshop. Periodontol 2000. 2015; 86(Suppl.):S56-S72

14. Papageorgakopoulos G, Greenwell H, Hill M, Vidal R, Scheetz J. Root coverage using acellular dermal matrix and comparing a coronally positioned tunnel to a coronally positioned flap approach. J Periodontol. 2008; 79:1022-30.

15. Ozenci I, Ipci SD, Cakar G, Yilmaz S. Tunnel technique versus coronally advanced flap with acellular dermal matrix graft in the treatment of multiple gingival recessions. J Clin Periodontol. 2015; 42:1135-1142. 